\title{
Pierre Lascoumes, Laure Bonnaud, Jean-Pierre Le Bourhis, Emmanuel Martinais, 2014, Le développement durable, une nouvelle affaire d'Etat?, Paris, PUF, 200 pages.
}

\section{Clémence Guimont}

\section{(2) OpenEdition}

\section{Journals}

Édition électronique

URL : http://journals.openedition.org/developpementdurable/11003

DOI : 10.4000/developpementdurable.11003

ISSN : 1772-9971

Éditeur

Association DD\&T

Référence électronique

Clémence Guimont, «Pierre Lascoumes, Laure Bonnaud, Jean-Pierre Le Bourhis, Emmanuel Martinais, 2014, Le développement durable, une nouvelle affaire d'Etat ?, Paris, PUF, 200 pages. », Développement durable et territoires [En ligne], Vol. 6, n² I Septembre 2015, mis en ligne le 30 septembre 2015 consulté le 24 septembre 2020. URL : http://journals.openedition.org/developpementdurable/11003 ; DOI : https://doi.org/10.4000/developpementdurable.11003

Ce document a été généré automatiquement le 24 septembre 2020.

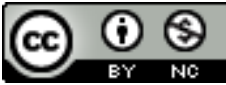

Développement Durable et Territoires est mis à disposition selon les termes de la licence Creative Commons Attribution - Pas d'Utilisation Commerciale 4.0 International. 


\section{Pierre Lascoumes, Laure Bonnaud, Jean-Pierre Le Bourhis, Emmanuel Martinais, 2014, Le développement durable, une nouvelle affaire d'Etat?, Paris, PUF, 200 pages.}

\section{Clémence Guimont}

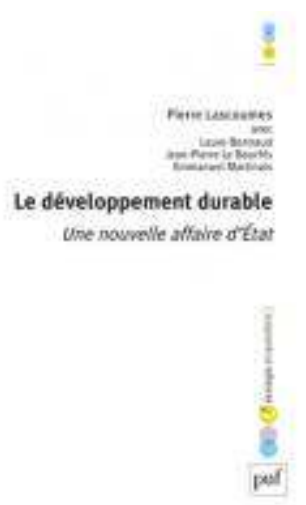

1 En 2007, le nouveau gouvernement Fillon affirme son intention de mettre en place un «New Deal écologique ». Il annonce aussi sa volonté de gouverner différemment l'environnement. Alors que le Grenelle de l'environnement est programmé et que la Révision générale des politiques publiques (RGPP) se prépare, un grand ministère de l'Ecologie est fondé, qui est marqué par la fusion des services des ministères de l'Ecologie, de l'Industrie et de l'Equipement. L'objectif est de faire travailler ensemble les différentes administrations de protection de l'environnement et les administrations qui soutiennent les activités économiques (notamment transports, industries et énergies), souvent issues des différents grands corps de l'Etat. C'est cette fusion entre services qui est étudiée par les auteurs, qui sont sociologues ou politistes et tous spécialistes de l'action publique environnementale. 
2 Pour comprendre les tenants et les aboutissants de cette fusion des administrations, les auteurs ont mené un large travail empirique avec plus de 70 entretiens et une analyse documentaire. Ils pointent dans cet ouvrage les trois difficultés principales que rencontre cette réforme : 1) les organisations et routines bien établies sont des obstacles à ce projet de transformation de l'Etat et des administrations ; 2) la diversité de pratiques et de mentalités, loin de l'administration monolithique fantasmée rend difficile la mise en œuvre de cette réforme ; 3) le rôle des individus et des relations interpersonnelles, au-delà de l'inertie et des résistances des institutions est déterminant pour assurer le changement de pratiques et réussir la réforme. Ces trois éléments permettent également de comprendre pourquoi la question environnementale est encore traitée de façon sectorielle, malgré la volonté politique d'en faire un problème transversal.

3 Pour présenter leurs résultats en détail, les auteurs proposent dans cet ouvrage une analyse séquentielle de cette réforme, allant de sa mise sur agenda à son évaluation.

4 Les trois premiers chapitres sont consacrés à la genèse de la fusion et à son articulation avec le Grenelle de l'environnement et la Révision générale des politiques publiques (RGPP). Si la proposition gouvernementale se nourrit de réflexions de la société civile, elle adapte aussi son projet d'après des logiques partisanes, administratives et bureaucratiques. Il y a au départ une volonté politique de la droite de construire un discours écologique qui lui est propre, en rappelant notamment que de nombreuses politiques publiques environnementales ont été mises en place sous des gouvernements de droite (comme la charte de l'environnement en 2006 et les grandes lois de protection dans les années 1970). Mais la mise en œuvre de cette fusion est aussi liée à la volonté de rationaliser et de diminuer le coût de l'action publique. Le contexte administratif est celui de la RGPP et de l'intériorisation par les hauts fonctionnaires de la nécessité de réduire le nombre de fonctionnaires. De plus, " cette fusion ministérielle avait pour but d'affaiblir le poids et l'influence des grands corps techniques au sein de l'administration. » (p. 43) Les différents corps de l'Etat entretiennent des luttes de pouvoir pour défendre leur réputation et leur prérogative, au détriment parfois de la mise en œuvre du développement durable. Ces différents corps subissent les transformations de l'action publique liées au New Public Management avec la promotion d'instruments ou de dispositifs décisionnels comme la gouvernance à cinq, la participation et la réduction du nombre de fonctionnaires.

5 Comment l'administration locale met-elle en place ce projet national ? Les trois chapitres suivants s'intéressent à la traduction territoriale de cette réforme avec la mise en place des Directions Régionales de l'Environnement et de l'Aménagement et du Logement (DREAL), issues de la fusion des services ministériels. On note des effets différenciés de la fusion sur la mise en œuvre des politiques publiques de l'environnement. Cela donne lieu à une grande pluralité de façons de construire les services déconcentrés avec des conceptions différentes et souvent peu opérantes du développement durable. Celles-ci ne se caractérisent pas par le corps, le genre professionnel ou le type de parcours. Les réponses sont très hétérogènes. Le développement durable n'est en général pas vu comme une finalité mais comme un cadre d'action et comme la justification d'une réorganisation en cours. L'organisation des DREAL dépend aussi fortement du territoire dans lequel le directeur arrive. Ces traductions locales disposent d'une large marge de manœuvre par rapport aux directives nationales. Elles varient en fonction donc du territoire. Elles offrent aussi de 
larges possibilités aux acteurs locaux et aux architectures institutionnelles, ce qui explique leur diversité et leur hétérogénéité.

6 L'ouvrage retrace de façon synthétique et efficace les différentes étapes de la fusion des services ministériels. L'analyse est d'autant plus convaincante qu'elle s'appuie sur un large travail empirique, leur permettant d'étayer leur démonstration avec de nombreuses citations d'entretiens. Les auteurs montrent les difficultés de faire du développement durable une politique transversale, tant la sectorisation est forte dans l'action publique française. L'analyse de trajectoires des agents des administrations locales et la description des traductions locales de la fusion des services permettent d'acquérir une bonne compréhension des mécanismes de mise en œuvre des politiques territoriales.

7 On peut toutefois regretter quelques flous dans l'usage de certaines notions. Ainsi, la notion de New Public Management (NPM), présente à travers la mention de la RGPP et de la contrainte financière notamment, n'est pas abordée de front. Alors que le NPM est mentionné tout au long de l'analyse séquentielle, on ne comprend pas ses implications, tant dans les représentations des agents que dans leurs pratiques. Des précisions auraient été utiles afin de saisir ses caractéristiques et ses conséquences sur les méthodes et pratiques de l'action publique (nationale et locale).

8 La notion de développement durable nous paraît également lacunaire. En effet, les auteurs évincent l'analyse des effets des réformes décrites sur la notion même de développement durable. Quelles sont les conséquences de l'appropriation du développement durable par une multiplicité d'intermédiaires et d'acteurs? S'il n'est plus à démontrer que le développement durable est vidé de son contenu ${ }^{1}$, il aurait été intéressant de décomposer ces mécanismes plus en profondeur pour bien saisir la spécificité des politiques publiques de développement durable.

9 Cette analyse très classique et presque routinière des politiques publiques est éclairante sur la genèse de cette fusion et les difficultés de sa mise en œuvre, notamment liées aux concurrences entre grands corps de l'Etat et aux effets différenciés de son application locale. Mais si elle mobilise efficacement les outils de théorie des politiques publiques, elle ne les interroge pas assez au regard des spécificités de « l'objet environnement ».

\section{NOTES}

1. Bruno Villalba (ed.), Appropriations du développement durable. Emergences, diffusions, traductions, Villeneuve d'Ascq, Presses Universitaires du Septentrion, 2009, 388 p. 


\section{AUTEUR}

\section{CLÉMENCE GUIMONT}

Clémence Guimont est doctorante en science politique au CERAPS (CNRS et Université Lille 2). 